\title{
6PPD-Quinone: Revised Toxicity Assessment and Quantification with a Commercial Standard
}

Zhenyu Tian, ${ }^{1,2 *}$ Melissa Gonzalez, ${ }^{1,2}$ Craig A. Rideout, ${ }^{1,2}$ Haoqi (Nina) Zhao, ${ }^{3}$ Ximin Hu, ${ }^{3}$ Jill

Wetzel, ${ }^{4}$ Emma Mudrock, ${ }^{4}$ C. Andrew James, ${ }^{1,2}$ Jenifer K. McIntyre, ${ }^{4,5}$ Edward P Kolodziej ${ }^{1,2,3 *}$

${ }^{1}$ Center for Urban Waters, Tacoma, WA, 98421, USA.

${ }^{2}$ Interdisciplinary Arts and Sciences, University of Washington Tacoma, Tacoma, WA, 98421, USA.

${ }^{3}$ Department of Civil and Environmental Engineering, University of Washington, Seattle, WA, 98195, USA.

${ }^{4}$ Washington Stormwater Center, Puyallup Research \& Extension Center (PREC), Puyallup, WA 98371, USA.

${ }^{5}$ School of the Environment, Washington State University, PREC, Puyallup, WA 98371, USA.

\section{Supporting information}

10 Pages, 3 Tables, and 4 Figures

December 8, 2021

Manuscript in preparation for submission to:

Environmental Science \& Technology Letters

*Co-corresponding author contact information

Email: z.tian@northeastern.edu,koloj@uw.edu

Mail: Center for Urban Waters

326 East D Street, Tacoma, WA 98421 


\section{In-house standards in the previous work}

For clarity and transparency, this part provides a brief summary of the source and use of in-house 6PPD-Q standards. In the previous work (Tian et al, 2021), two in-house standards of 6PPDquinone were used: one purified from tire wear particle (TWP) leachate, and one purified from 6PPD ozonation. Both standards went through the sequential fractionation with $\mathrm{C} 18$, PFP, and phenyl HPLC columns, and the tire-derived 6PPD-Q standard has more pre-separation steps such as silica gel and ion exchange chromatography. Further details of fractionation and synthesis method were recorded in the supporting materials of the previous work. Both tire-derived and ozonated standards were used for exposure experiments because the tire-derived standard was limited. All the quantification were based on the calibration curves made from tire-derived 6PPDQ standard (blue curves in Figure S1), as it was thought to be purer and more consistent. All the comparisons with the HPC commercial standard were also based on this in-house standard.

\section{Water sample extraction method}

For accurate quantification, duplicate samples or blanks were first spiked with D5-6PPD-Q (25 ng) extracted by an established method with modifications. To prevent clogging, $0.5 \mathrm{~g}$ pre-cleaned micro glass beads (Filter Aid 400, 3M, MN) were added to solid phase extraction (SPE) cartridges (200 mg, 6 mL Oasis HLB, Waters, MA). SPE cartridges were preconditioned (5 mL methanol, $25 \mathrm{~mL}$ DI water; 5-10 mL/min). Water samples $(200 \mathrm{~mL})$ were loaded onto SPE cartridges at a rate of 5-10 mL/min. Cartridges were rinsed with $10 \mathrm{~mL} \mathrm{DI}$ water, dried $10 \mathrm{~min}$, and eluted with methanol (four $2.5 \mathrm{~mL}$ aliquots). Eluates were concentrated to $1 \mathrm{~mL}$ under $\mathrm{N}_{2}$ and transferred into autosampler vials. Method blanks were extracted identically and used for quality control. Extracts were stored at $-20{ }^{\circ} \mathrm{C}$ until instrumental analysis. 


\section{Instrumental analysis}

For the LC-MS/MS analysis, chromatography used a reverse phase C18 column (Agilent Poroshell HPH-C18 $2.1 \times 100 \mathrm{~mm}, 2.7 \mu \mathrm{m}$ particle size) and C18 guard column at $45^{\circ} \mathrm{C}$, injection volume $5 \mu \mathrm{L}$, flow rate $0.2 \mathrm{~mL} / \mathrm{min}$, and binary gradient of $0.1 \%$ formic acid in each of water (A) and methanol (B): [50\% B 0-0.5 min, 50\%-100\% B 0.5-10.5 min, 100\% B 10.5-12 $\mathrm{min}, 100 \%-50 \%$ B 12-13 $\mathrm{min} ; 50 \%$ B 13-15 $\mathrm{min}]$. Detection used electrospray ionization (ESI + ) and multi reaction monitoring $(\mathrm{MRM})$ modes Different gas temperatures $\left(250,300,350{ }^{\circ} \mathrm{C}\right)$, capillary voltages $(2500,3000,3500 \mathrm{~V})$, and fragmentor voltages $(90-150 \mathrm{~V})$ were tested in the method development process, and the ones with higher 6PPD-Q responses were selected. The optimized instrument parameters are listed in Table S1. According to MS/MS data from HRMS and MRM screening, $m / z \quad 299 \rightarrow 215(14 \mathrm{eV})$ and $299 \rightarrow 187(26 \mathrm{eV})$ were selected as the qualitative and quantitative ion transitions, respectively. 6PPD-Q concentrations were estimated from a sevenpoint calibration curve $(0.025-50 \mu \mathrm{g} / \mathrm{L}$, Figure S2) with D5-6PPD-Q as the isotopic internal standard (25 $\mu \mathrm{g} / \mathrm{L}$, identical to sample extracts) for response normalization; determination coefficients $\left(\mathrm{R}^{2}\right)$ were $>0.995$.

\section{Spike recoveries}

All the spiked samples were extracted and analyzed in technical duplicates. The lower concentration $(5 \mathrm{ng} / \mathrm{L})$ spike experiment was repeated three times independently and the higher concentration $(50 \mathrm{ng} / \mathrm{L})$ was performed once, representing potential baseflow and storm conditions. Absolute recoveries were calculated by comparing peak areas in the samples spiked before and after extraction; relative recoveries were calculated by comparing measured and spiked concentrations. 
In the three low concentration spike tests, 6PPD-Q absolute recoveries were 58\%, 95\%, and 72\%. In the higher concentration spike test, the absolute recovery was $65 \%$. The absolute recovery of the internal standard (D5-6PPD-Q) was 34\%-82\% across different batches samples (not necessarily from spike tests, as all the samples were spiked with the internal standard). The lower absolute recoveries were caused by matrix suppression of the creek water samples, not the loss during SPE process. The relative recoveries of 6PPD-Q (i.e., the results corrected by internal standard) was $89 \%-116 \%$.

\section{UV-Vis measurements of 6PPD-Q standards}

The in-house standard (tire leachate derived) used for the quantification in the previous work (Tian et al 2021) was diluted in ethanol for the UV-Vis measurement. For comparison, stock solution was prepared from HPC standard 6PPD-Q solid with ethanol. All the measurement blanks and baselines were prepared by EtOH blanks. The UV absorption spectra agreed on the $360 \mathrm{~nm}$ peak for 6PPDQ, but the HPC standard was quantitative stronger at the same concentrations. We adjusted the absorbance to similar levels and evaluate the nominal/actual concentrations of the inhouse 6PPD-Q standard. When having the same actual concentrations $(0.95 \mathrm{mg} / \mathrm{L}$, comparing with the HPC standard), the nominal concentration of the in-house stock solution was $15 \mathrm{mg} / \mathrm{L}$ from our previous estimation (Figure S3). Therefore, this indicated that the actual 6PPD-Q concentrations of the in-house stocks were about $1 / 15$ of their nominal concentrations.

\section{Toxicity comparison rationale}

6PPD-quinone is among the most toxic chemicals known to aquatic life, along with certain pesticides and some metals. To evaluate relative acute toxicities of chemicals, we can compare their median lethal concentration (LC50) - the concentration that results in mortality for half of 
the test organisms in standardized toxicity tests. Smaller LC50 values indicate more toxic chemicals because less chemical is needed to cause $50 \%$ mortality.

The U.S. Environmental Protection Agency (EPA) Office of Pesticide Programs categorizes acute toxicity of chemicals to aquatic life in a range from 'practically non-toxic', with chemical effects at $>100 \mathrm{ppm}$, to 'very highly toxic', causing mortality at $<0.1 \mathrm{ppm}(<100 \mathrm{ppb})$.

The EPA has established aquatic life criteria (ALC) for toxic chemicals that designate a concentration deemed 'safe' for aquatic organisms if not exceeded over a fixed period of time and frequency (e.g., acute criteria are for $1 \mathrm{~h}$ every 3 years). ALC to protect against acute effects are typically based on concentrations that cause mortality for the four most sensitive species tested.

While there is not yet ALC for 6PPD-quinone, we can compare the LC50 for coho exposed to 6PPD-quinone with that of the most sensitive test organisms used to derive ALC. Among the 'very highly toxic' chemicals for which we have ALC, the toxicity of 6PPD-quinone is similar to that of the most toxic of 12 chemicals, all with LC50s $<1 \mathrm{ppb}$. This group is comprised of neurotoxic organophosphates (parathion, guthion, chlorpyrifos, diazinon, malathion) and organochlorines (mirex, endrin, DDT, methoxychlor, dieldrin, toxaphene), as well as cadmium (Table 1). Acute ALC exist for an additional 21 chemicals including many metals that are also 'very highly toxic' (causing mortality at $<100 \mathrm{ppb}$ ), but their lowest LC50s are $>1 \mathrm{ppb}$ and therefore much less toxic than 6PPD-quinone (LC50 for coho salmon $\sim 0.1 \mathrm{ppb}$ ).

\section{References for Toxicity Comparison:}

US EPA, Technical Overview of Ecological Risk Assessment - Analysis Phase: Ecological Effects Characterization https://www.epa.gov/pesticide-science-and-assessing-pesticiderisks/technical-overview-ecological-risk-assessment-0 (accessed 2022-01-04).

US EPA, Supplemental Module: Aquatic Life Criteria https://www.epa.gov/wqstech/supplemental-module-aquatic-life-criteria (accessed 2022-01-04). 
US EPA, National Recommended Water Quality Criteria - Aquatic Life Criteria Table https://www.epa.gov/wqc/national-recommended-water-quality-criteria-aquatic-lifecriteria-table (accessed 2022-01-04).

Table S1. Exposure concentrations and observed mortality data of juvenile coho salmon experiments using the commercial HPC 6PPD-Q standard. All negative control fish survived and did not show any symptom ( $\mathrm{N}=24,6$ in range finding and 18 in definitive exposure).

\begin{tabular}{|c|c|c|c|c|c|}
\hline Batch & Nominal conc. (ng/L) & Measured conc. (ng/L) & $\%$ Conc. error ${ }^{a}$ & Mortality ratio & Mortality \% \\
\hline 0 & 20.0 & $23.1^{\mathrm{b}}$ & & $0 / 6$ & $0 \%$ \\
\hline 0 & 35.6 & $41.1^{\mathrm{b}}$ & & $0 / 6$ & $0 \%$ \\
\hline 0 & 63.2 & $73.0^{\mathrm{b}}$ & & $0 / 6$ & $0 \%$ \\
\hline 0 & 112.5 & $130^{\mathrm{b}}$ & & $4 / 6$ & $67 \%$ \\
\hline 0 & 200.0 & $231^{b}$ & & $6 / 6$ & $100 \%$ \\
\hline 1 & 50 & 53.2 & $6 \%$ & $0 / 6$ & $0 \%$ \\
\hline 1 & 66 & 68.9 & $4 \%$ & $1 / 5$ & $20 \%$ \\
\hline 1 & 87 & 97.1 & $12 \%$ & $4 / 6$ & $67 \%$ \\
\hline 1 & 115 & 122 & $6 \%$ & $4 / 6$ & $67 \%$ \\
\hline 1 & 152 & 162 & $7 \%$ & $5 / 6$ & $83 \%$ \\
\hline 1 & 200 & 226 & $13 \%$ & $5 / 6$ & $83 \%$ \\
\hline 2 & 60 & 68.1 & $14 \%$ & $0 / 6$ & $0 \%$ \\
\hline 2 & 72 & 83.5 & $16 \%$ & $2 / 6$ & $33 \%$ \\
\hline 2 & 86 & 99.3 & $15 \%$ & $2 / 5$ & $40 \%$ \\
\hline 2 & 104 & 115 & $10 \%$ & $4 / 6$ & $67 \%$ \\
\hline 2 & 125 & 154 & $22 \%$ & $3 / 6$ & $50 \%$ \\
\hline 2 & 150 & 194 & $29 \%$ & $5 / 6$ & $83 \%$ \\
\hline 3 & 60 & 70.5 & $17 \%$ & $3 / 6$ & $50 \%$ \\
\hline 3 & 72 & 84.6 & $17 \%$ & $5 / 6$ & $83 \%$ \\
\hline 3 & 86 & 105 & $22 \%$ & $5 / 5$ & $100 \%$ \\
\hline 3 & 104 & 124 & $19 \%$ & $4 / 5$ & $80 \%$ \\
\hline 3 & 125 & 151 & $21 \%$ & $4 / 6$ & $67 \%$ \\
\hline \multirow[t]{3}{*}{3} & 150 & 190 & $27 \%$ & $4 / 6$ & $67 \%$ \\
\hline & & average & $15 \%$ & & \\
\hline & & median & $16 \%$ & & \\
\hline
\end{tabular}

a. The measured concentrations were systematically higher than the nominal concentrations (median 16\%), potentially caused by the volumetric vessels (Hamilton syringes and glass pipettes).

b. Quantification method was not finalized by the time of batch 0 , so the measured concentrations were back calculated according to the median error of batch 1-3. 
Table S2. Instrumental parameters for quantification of 6PPD-Q and D5-6PPD-Q by LC-MS/MS.

\begin{tabular}{ll}
\hline Parameter & Value \\
\hline Gas temp & $300^{\circ} \mathrm{C}$ \\
Gas flow & $5 \mathrm{~L} / \mathrm{min}$ \\
Sheath gas temp & $400^{\circ} \mathrm{C}$ \\
Sheath gas flow & $11 \mathrm{~L} / \mathrm{min}$ \\
Capillary voltage & $3,000 \mathrm{~V}$ \\
Nozzle voltage & $500 \mathrm{~V}$ \\
Nebulizer & $45 \mathrm{psi}$ \\
Fragmentor voltage & $110 \mathrm{~V}$ \\
\hline
\end{tabular}

Table S3. Instrumental parameters for mass spectrometry detection of 6PPD-Q and D5-6PPD-Q.

\begin{tabular}{lrrrl}
\hline Compound & precursor ion $(\mathbf{m} / \mathbf{z})$ & fragment ion $(\mathbf{m} / \mathbf{z})$ & fragmentation energy $(\mathbf{V})$ & Function \\
\hline 6PPD-Q & 299.2 & 215.1 & 14 & qualifier \\
6PPD-Q & 299.2 & 187.1 & 26 & quantifier \\
D5-6PPD-Q & 304.2 & 220.1 & 14 & qualifier \\
D5-6PPD-Q & 304.2 & 192.1 & 26 & quantifier \\
\hline
\end{tabular}



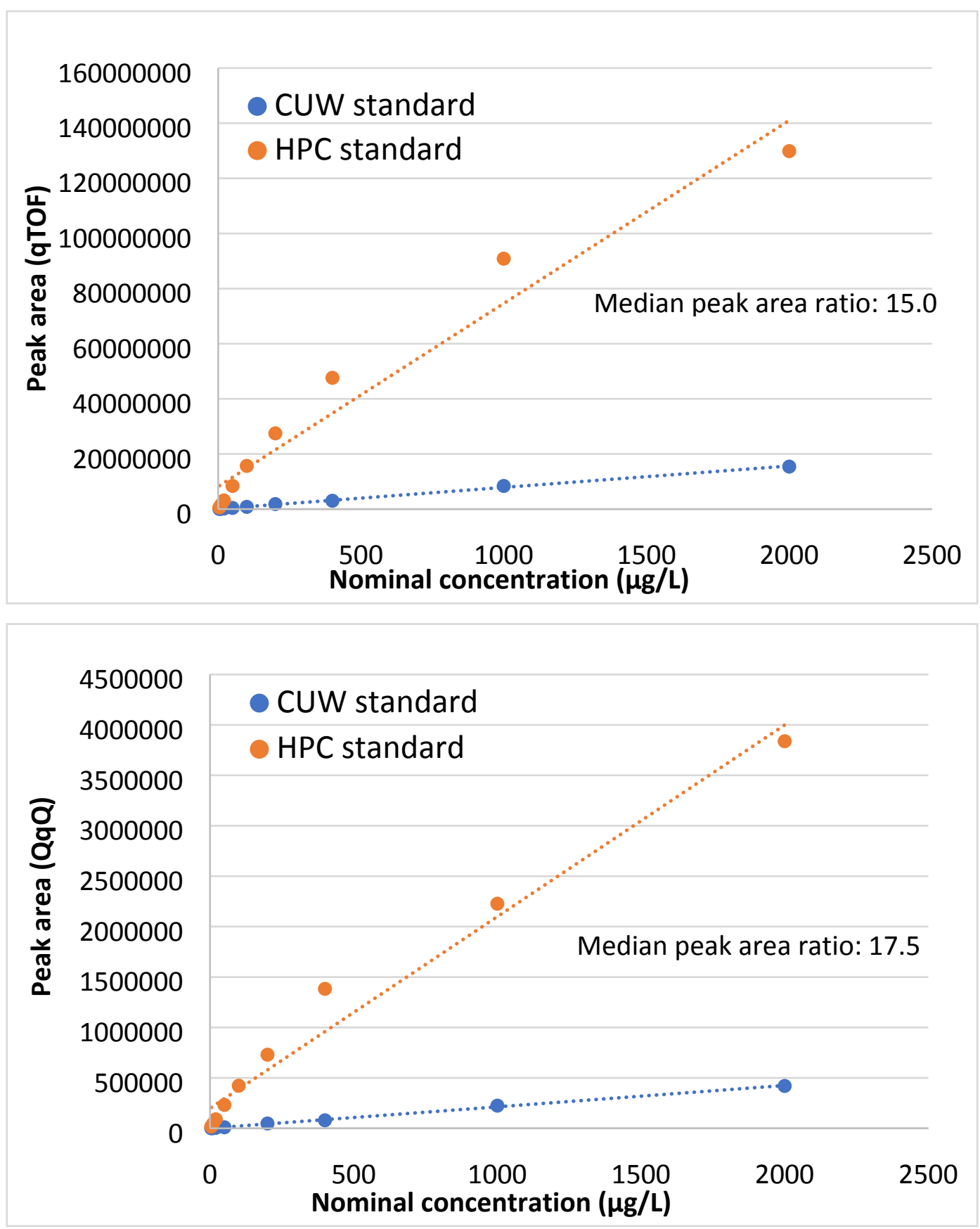

Figure S1. Observed peak area response curves of commercial ("HPC standard") and in-house tire derived ("CUW standard") 6PPD-Q standards measured by (A) UHPLC-qTOF-HRMS and (B) HPLC-QqQ-MSMS. HPC standard reached saturation at these concentrations (100-2000 $\mu \mathrm{g} / \mathrm{L}$ in methanol), but these were not the ranges used in the isotope dilution method (see Figure S2 below). 


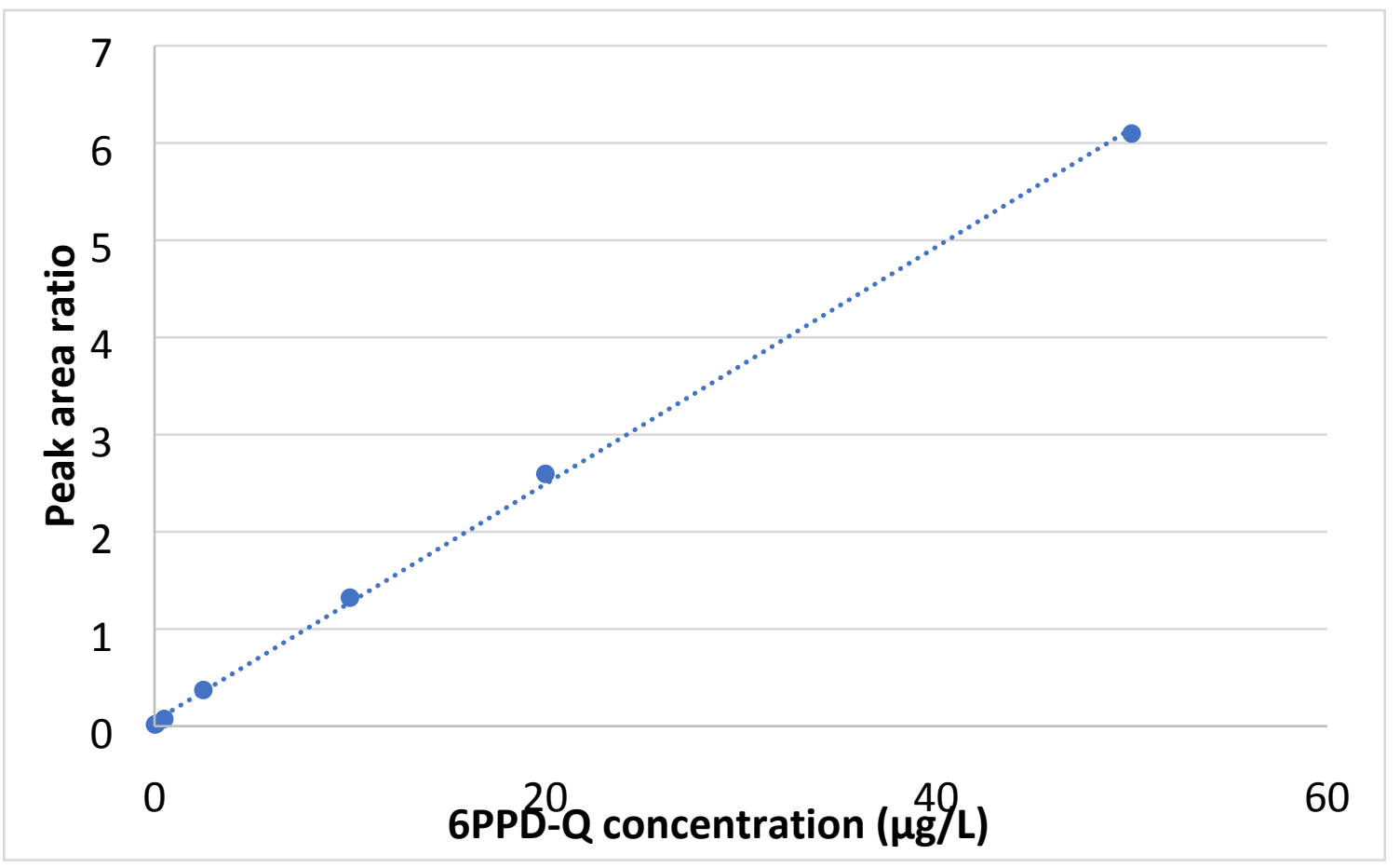

Figure S2. 7-point standard calibration curve used in the isotope dilution method. Y-axis

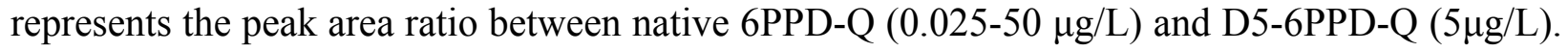
The linear range would end between $50 \mu \mathrm{g} / \mathrm{L}$ and $100 \mu \mathrm{g} / \mathrm{L}$.

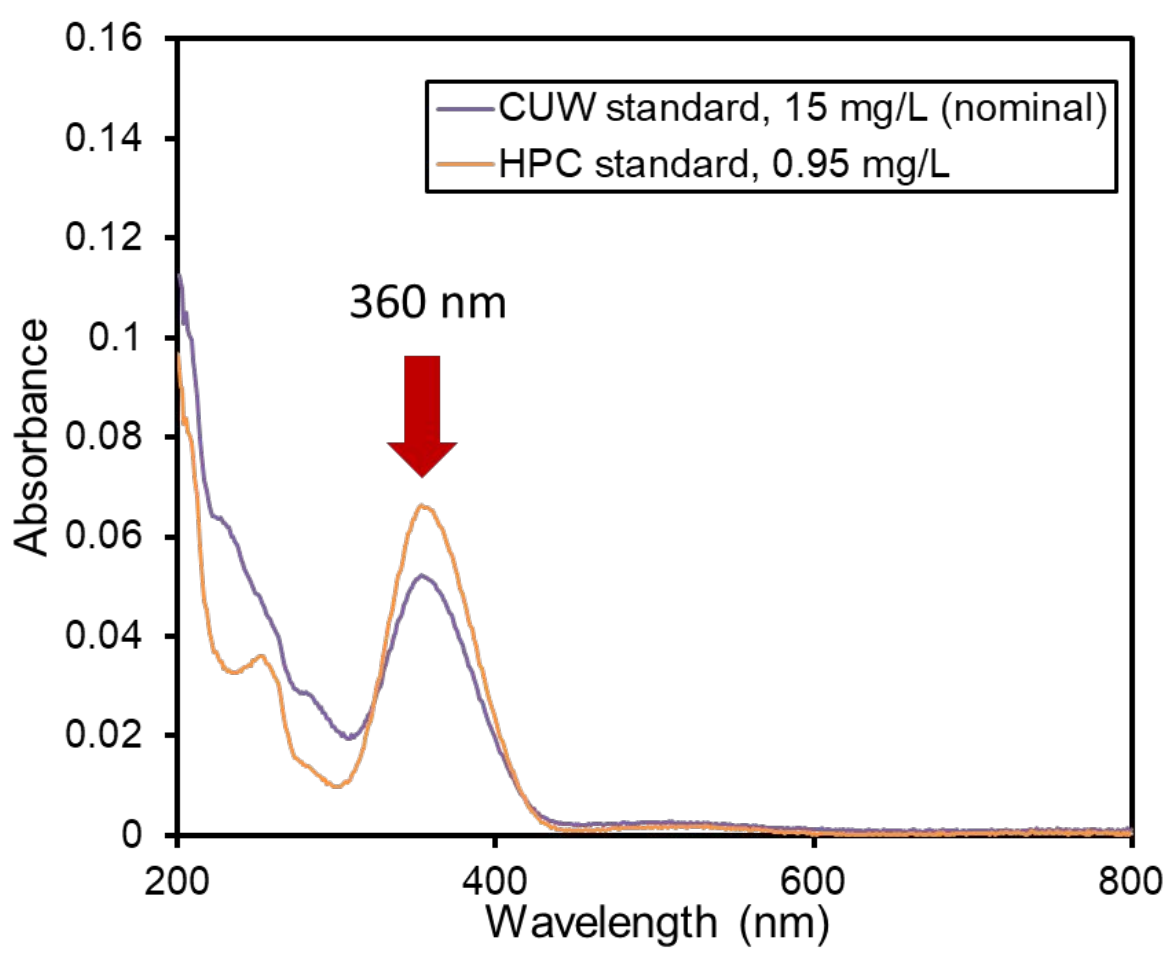

Figure S3. UV-Vis spectra of the tire leachate derived 6PPD-Q (CUW standard, purple line) and HPC 6PPD-Q standard (orange line). Both the 6PPD-Q stocks have actual concentrations of 0.95 $\mathrm{mg} / \mathrm{L}$. The nominal concentration of the CUW was $15 \mathrm{mg} / \mathrm{L}$, which was overestimated. 

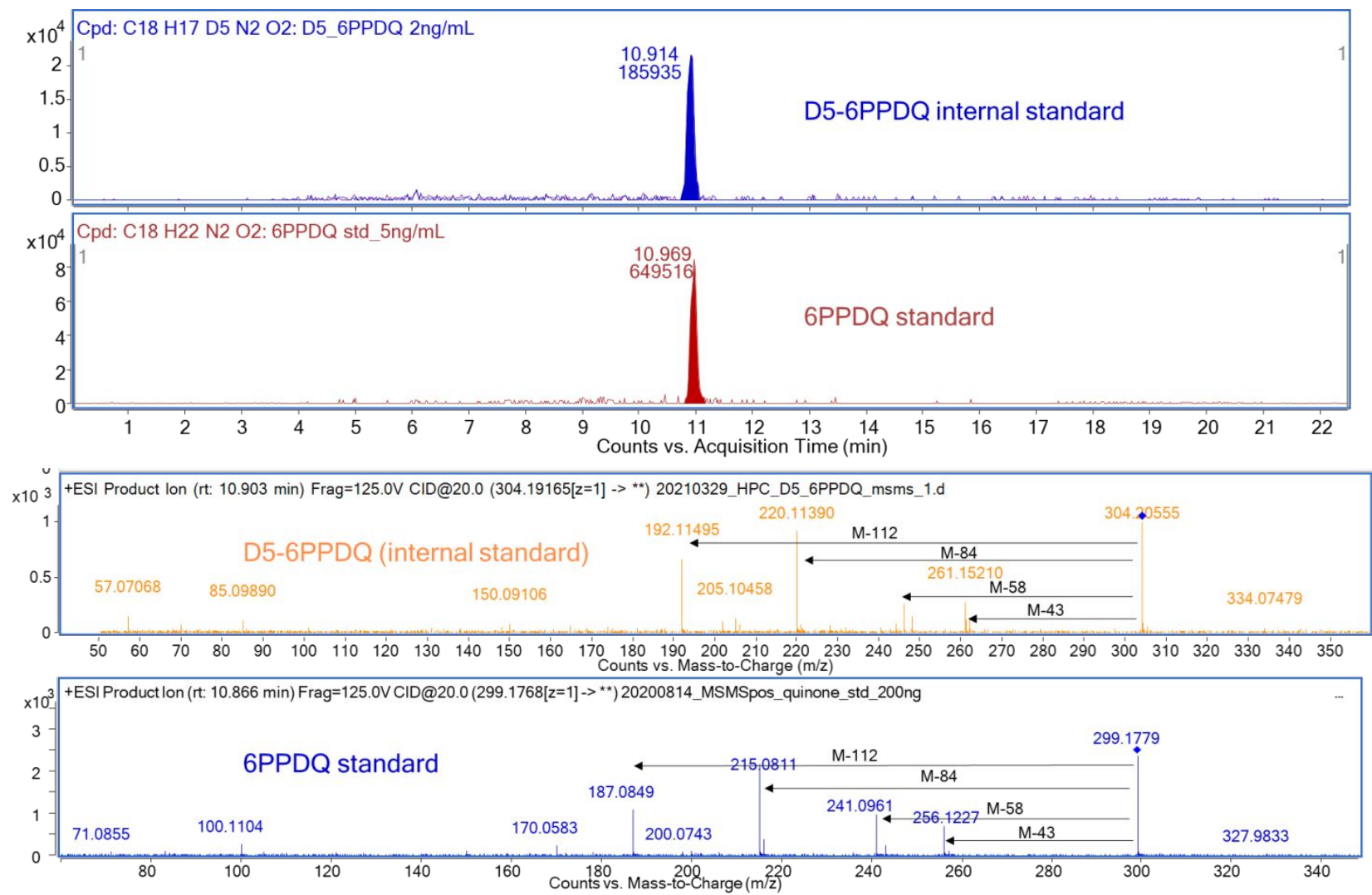

Figure S4. Chromatograms and MS/MS spectra of D5-6PPD-Q and 6PPD-Q commercial standards, analyzed by UPLC-qTOF-HRMS. Deuterated (D5-) and native 6PPD-Q have the same fragmentation patterns (M-43, M-58, M-84, M-112). 